\title{
EFFECT OF NICKEL OXIDE ON THE CONDUCTIVITY OF POLYMER BLEND ELECTROLYTE DOPED WITH SODIUM IODIDE AND ITS APPLICATION IN DYE-SENSITIZED SOLAR CELL
}

\author{
Norshahirah M. Saidi, Fatin Saiha Omar, K. Ramesh and S. Ramesh* \\ Centre for Ionics University of Malaya, Department of Physics, Faculty of Science, University of Malaya, \\ 50603 Kuala Lumpur, Malaysia. \\ *Correspondence Author: ramesh@um.edu.my,norshahirahsaidi@gmail.com,ftnsaiha@gmail.com, \\ rameshkasi@um.edu.my \\ Received: $13^{\text {th }}$ Aug $2018 \quad$ Reviewed: $11^{\text {th }}$ Feb $2019 \quad$ Accepted: $11^{\text {th }}$ Feb 2019
}

DOI: https://doi.org/10.22452/mjs.vol38no1.1

\begin{abstract}
Ionic conductivity of electrolyte is one of the factor that influences the efficiency of dye-sensitized solar cell (DSSC). Herein, gel polymer electrolyte (GPE) consist of polyacrylonitrile (PAN), poly(1-vinylpyrrolidone-co-vinyl acetate) (P(VP-co-VAc)), sodium iodide $(\mathrm{NaI})$ dopant and nickel oxide $(\mathrm{NiO})$ additive was prepared using solution casting method. The ionic conductivity study revealed that after the inclusion of $\mathrm{NiO}$, the conductivity was increased from $2.33 \mathrm{mS} \mathrm{cm}^{-1}$ (for polymer blend) to $4.84 \mathrm{mS} \mathrm{cm}^{-1}$. Besides the dielectric properties, the electrical modulus study was conducted to analyse the effect of electrode polarization and ionic conductivity of the GPE. Under the exposure of AM 1.5, the fabricated DSSC utilizing the prepared GPE exhibited photoenergy conversion efficiency of $3.98 \%$ with a short circuit current density $\left(\mathrm{J}_{\mathrm{SC}}\right)$ of $15.03 \mathrm{~mA} \mathrm{~cm}^{-2}$, open circuit voltage $\left(\mathrm{V}_{\mathrm{OC}}\right)$ of $0.57 \mathrm{mV}$ and fill factor $(\mathrm{FF})$ of 44.0 $\%$.
\end{abstract}

Keywords: Gel polymer electrolyte, Dye-sensitized solar cell, Nanofiller, NiO, Polyacrylonitrile

\section{INTRODUCTION}

Transition from fossil fuel to renewable energy sources is one of the best eco-friendly options to mitigate the emission of greenhouse gasses. Among all renewable energy sources, solar energy is the most feasible way to be utilized to support the production of electricity. In the past few years, dyesensitized solar cells (DSSCs) have received tremendous attention for renewable energy exploration. Compared to silicon solar cells, DSSC offer relatively cheap, environmentally benign, tuneable optical properties and good photocurrent conversion efficiency. DSSC have been found can work best under low flux of sunlight due to the fact that the best DSSC (employing liquid electrolyte) can convert up to $28 \%$ of slower pace of light energy into electricity (N.K.A. Hamed et.al., 2017; M. Rasheduzzaman et.al., 2016).

Commonly, the desirable electrolyte for DSSC must have excellent ionic conductivity $\left(10^{-2}-10^{-3}\right.$ $\mathrm{S} / \mathrm{cm})$, low melting point $\left(<-20^{\circ} \mathrm{C}\right)$, high boiling point $\left(>100^{\circ} \mathrm{C}\right)$, low viscosity to favour high diffusion coefficient of redox mediator, minimized charge carrier transport resistance, good compatibility with electrodes, non-toxic and chemically stable (P.S. Dhapola 
et.al., 2018; J. Wu et.al., 2015). Even though liquid electrolyte can guarantee high ionic conductivity, there are some issues which prevent DSSC with liquid based electrolyte from further commercialization such as leakage while sealing and volatilization of the solvent (P. Nijisha et.al., 2016). Therefore, gel polymer electrolytes (GPEs) have been much studied to replace liquid electrolyte.

Many polymers have been used as host polymers for the preparation of GPE such as polyacrylonitrile (PAN), poly(ethylene oxide) (PEO), poly(methyl methacrylate) (PMMA) etc. Out of the aforesaid polymers, PAN is undoubtedly a good potential polymer mainly owing to its dominant polar nitrile groups. Nitrile groups in PAN is advantageous as it possesses a high dipole moment (3.9 D) which can hold more free cations. Moreover, PAN can dissolve easily in polar organic solvents (e.g. dimethyl sulfonate (DMSO), ethylene carbonate (EC), propene carbonate (PC) and dimethylformamide (DMF)) and turn into gel form by trapping the polar solvent molecules in their polymer matrix (Q.-Y. Wu et.al., 2012; A.K. Arof et.al., 2017). Nevertheless, PAN doesn't exhibit high ionic conductivity and good mechanical properties simultaneously which is not practical for DSSC application.

Various strategies have been experimented to acquire high conductivity and mechanical properties of GPE including crosslinking/blending/copolymerization of polymers as well as incorporation of additives (plasticizers, ceramic materials and ionic liquid). In this regard, poly(1vinylpyrrolidone-co-vinyl acetate) (P(VP-co-VAc)) co-polymer has been chosen to be blended with PAN. Because of the combination of two different structures; crystalline (PVP) and amorphous (PVAc), this co-polymer can supply plasticity and mechanical strength to GPE system. Meanwhile, nanostructured materials are widely known have large surface area which can form wide contact area with other substances and outstanding physical/mechanical properties. When nanostructured materials are introduced into GPE, they can be a cross-linking centers for the polymer segments and helps in impeding the crystallization tendency of the polymer chain. These mechanisms increases the amorphocity of the polymer system which can create more spaces to facilitate $\mathrm{I}^{-} / \mathrm{I}_{3}^{-}$diffusion and elevate the ionic conductivity.

In the present study, PAN is blended with P(VP-co-VAc) and EC/PC mixture was used as the solvent as this mixture has polar groups which are available for dipole-dipole interaction with the nitrile groups of PAN. NaI salt and nickel oxide particles $(\mathrm{NiO})$ are further incorporated into PAN-P(VP-coVAc) blended system in order to boost the ionic conductivity and increase the efficiency of DSSC. The effect of the $\mathrm{NiO}$ addition on the conductivity properties of GPE as well as on the DSSC performance are studied.

\section{MATERIALS}

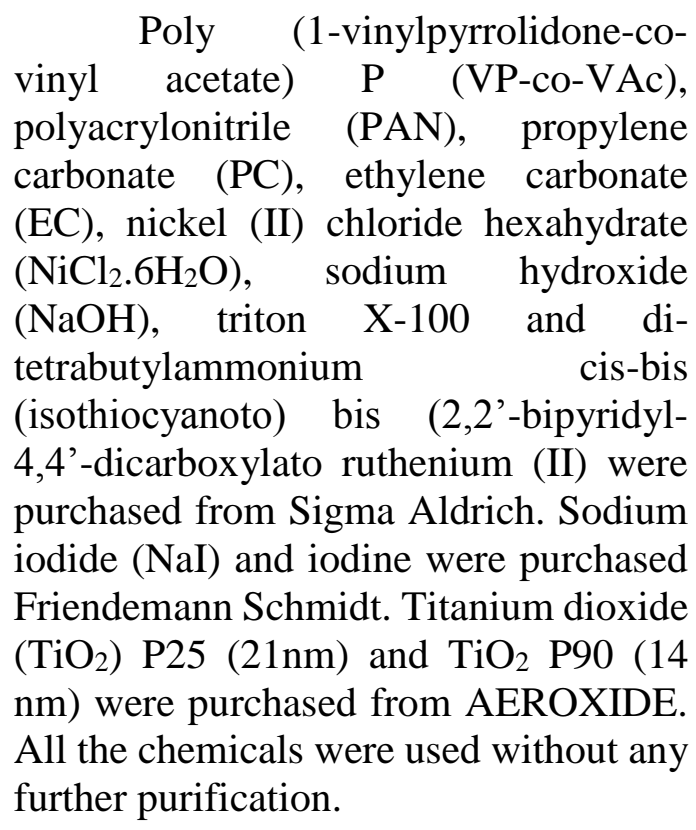




\subsection{Preparation and characterization of gel polymer electrolyte}

Host polymer employed with 1:1 w/w of PAN: P (VP-co-VAc) were dissolved in 1:1 w/w mixture of EC:PC solvent and labelled as PP. This experimental procedure was repeated in the second system by adding $40 \%$ of NaI salt and $\mathrm{I}_{2}$ (one tenth of the total amount of NaI used) and labelled as PPNa. For second system, $40 \%$ of sodium iodide salt and iodine were initially dissolved in the solvent containing 1:1 w/w of EC:PC solvent and stirred at room temperature to ensure the homogenous mixture of $\mathrm{NaI}$ and $\mathrm{I}_{2}$. Then, the mixture was added into $1: 1 \mathrm{w} / \mathrm{w}$ of host polymer PAN/ $\mathrm{P}$ (VP-co-VAc) and stirred at $100^{\circ} \mathrm{C}$. The electrolyte was cooled down to room temperature after the electrolyte was fully dissolved. For third system, the same steps from the second system was repeated except $5 \mathrm{wt} . \%$ of $\mathrm{NiO}$ particles were added into the GPE (PPNa) and sonicated for 30 minutes to make sure the particles are well dispersed within the prepared GPE. Th GPE for the third system was denoted as PPNaNiO.

The ionic conductivity of formulated GPE was determined using Cole-Cole plot electrochemical impedance spectroscopy (EIS, Hioki LCR Hi-Tester Model 3532-50) at room temperature using an $\mathrm{AC}$ voltage of 10 $\mathrm{mA}$ within a frequency range of $50 \mathrm{~Hz}$ to $5 \mathrm{MHz}$. All the formulated GPE were sandwiched between two stainless steel blocking electrodes. The temperature dependent EIS studies were also carried out on the formulated GPE from $30^{\circ} \mathrm{C}$ to $100^{\circ} \mathrm{C}(303 \mathrm{~K}$ to $373 \mathrm{~K})$.

\subsection{Synthesis of nickel oxide (NiO)}

$\mathrm{NiO}$ was synthesized according to our reported literature (N. Duraisamy et.al., 2016). $1 \mathrm{M}$ of $\mathrm{NiCl}_{2} \cdot 6 \mathrm{H}_{2} \mathrm{O}$ was dissolved in $50 \mathrm{~mL}$ of deionized water under constant stirring for 10 minutes. Then, the solution was subjected to the sonication using an ultrasonic probe. At the same time, $2 \mathrm{M}$ of $\mathrm{NaOH}$ was added drop wise into the above solution. The entire chemical reaction was carried out under constant amplitude of $60 \%$ for one hour. During this process, the ultrasonic irradiation was conducted without any coolant, so the temperature reached about $60 \pm 5^{\circ} \mathrm{C}$. The colloidal mixture then washed for 5-6 times with deionized water using centrifugation. The precipitate obtained was then calcined at $250^{\circ} \mathrm{C}$ for 3 hours in an electric furnace.

\subsection{Fabrication and characterization of dye-sensitized solar cell}

All the developed GPE samples were assembled in the DSSC devices by sandwiching them in between of photoanode and platinum electrode. The photovoltaic performance of the samples were studied under the illumination of $100 \mathrm{~mW} \mathrm{~cm}^{-2}$ simulated sunlight, from a Newport LCS-100 Series solar simulator, with a Metrohm Autolab potentiostat (PGSTAT128N). The electrochemical impedance spectroscopy (EIS) studies of the fabricated DSSC also were done under frequency in the range of $50 \mathrm{~Hz}$ to 5 $\mathrm{MHz}$ and $10 \mathrm{mV}$ of the AC voltage. The impedance spectra that were obtained were fitted using Metrohm Autolab Nova software. The electrochemical equivalent circuit is illustrated as the inset of Fig. 1(b) was used to attain the best numerical fit for the EIS results. 


\section{RESULTS AND DISCUSSION}

\subsection{EIS studies}

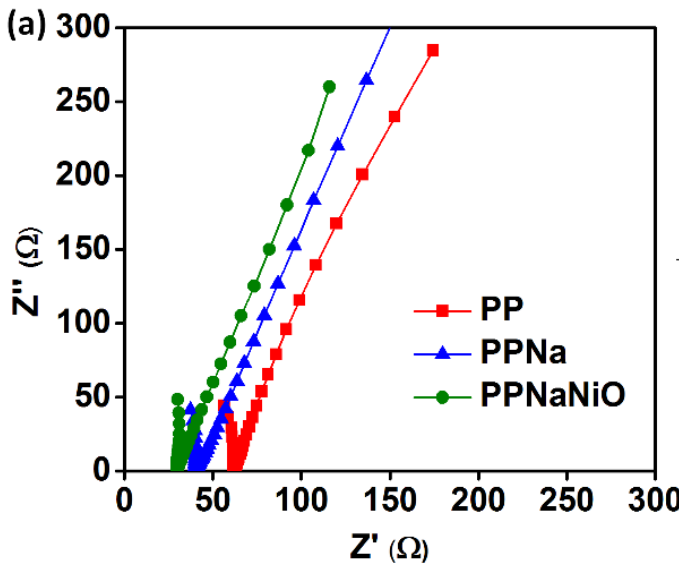

(b)

Figure 1. Cole-Cole plots of PP, PPNa and PPNaNiO.

The ionic conductivity $(\sigma)$ of PP, $\mathrm{PPNa}$ and $\mathrm{PPNaNiO}$ at room temperature can be determined from the Cole-Cole plots of electrochemical impedance spectra (EIS) studies as shown in Figure 1. Typically, Cole-Cole plots demonstrated an impedance spectra of the samples that were sandwiched between two blocking electrode; stainless steel electrode. Cole-Cole plot can be divided into two regions; a semi-circle at high frequency range and a spike at low frequency range. The semi-circle corresponds to the bulk properties and the spike can be ascribed to the electrode polarization effect. The bulk resistance $\left(R_{b}\right)$ was obtained from the intersection of the semicircle at high frequency with the real axis in the complex impedance plots. The graph shows that $\mathrm{PPNaNiO}$ has the smallest $R_{b}$ value $(29.93 \Omega)$ as compared to PP $(62.13 \Omega)$ and $\mathrm{PPNa}$ $(40.83 \Omega)$. From the result, it is confirmed that the addition of $\mathrm{NaI}$ and $\mathrm{NiO}$ have contributed to more efficient of charge carrier transportation within the host polymer.

\subsection{Ionic conductivity studies}

(a)

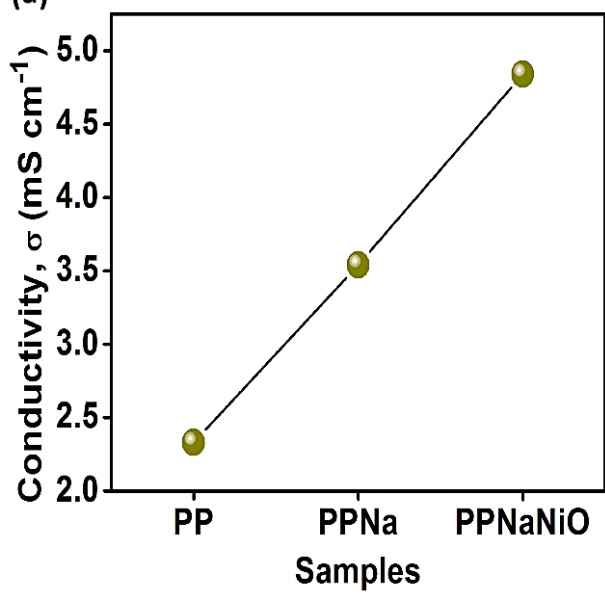

(b)

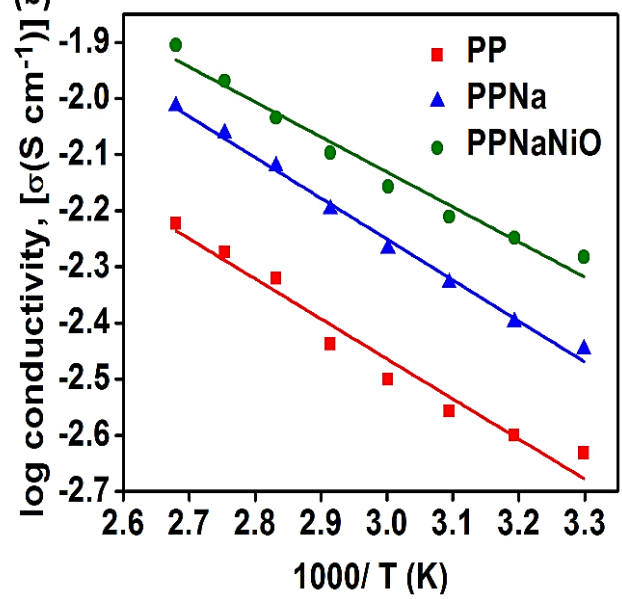

Figure 2. (a) The variation of ionic conductivity of PP, PPNa and PPNaNiO (b) the plot of logarithmic ionic conductivities versus 1000/T 
The $R_{b}$ values were then used to calculate the ionic conductivity $(\sigma)$ of the polymer electrolyte systems using the following equation:

$$
\sigma=t / A R_{b}
$$

Where $R_{b}$ : the thickness of the film, and $A$ : the area of the sample. From the ionic conductivity results (Figure 2 (a)), it can be seen that the conductivity increases with the addition of $\mathrm{NaI}$ in the polymer blend from 2.33 to $3.54 \mathrm{mS} \mathrm{cm}-1$ and further addition of $\mathrm{NiO}$ leads to enhancement with the maximum value of $4.84 \mathrm{~S} \mathrm{~cm}^{-1}$. The phenomenon of ionic conductivity can be explained by using the following ionic conductivity equation:

$$
\sigma(T)=\sum_{i} n_{i} q_{i} \mu_{i}
$$

Where $n$ indicates the density of the charge carriers, $q$ is the charge of ions and $\mu$ is the mobility of the ions. The conductivity exhibited by PP and the improvement in conductivity shown by PPNa and PPNaNiO can be correlated with several factors; (i) Decrease in polymer viscosity due to the dissolution of salt. Host polymer (PP) become less viscous after the dissolution of $\mathrm{NaI}$ (separation of $\mathrm{Na}^{+}$cations from $\mathrm{I}^{-}$ anions) which created more free charge carriers (V. Sundararajan et.al., 2017), (ii) the addition of $\mathrm{NiO}$ aid to further enhance the degree of dissociation of $\mathrm{NaI}$ salt by decreasing the solvation of the sodium ions. As a result, this boosts the dissociation of ions pairs and increase the ionic conductivity of GPE (N.S. Ramesh et.al., 2015), and (iii) the inclusion of $\mathrm{NiO}$ can reduce the interaction among the polymer chains and provide more pathways for mobility charge carriers throughout the electrolyte systems (L. Deanship, 2016).

The relationship between conductivity $(\sigma)$ and temperature $(1000 / T)$ for PP, PPNa and PPNaNiO are demonstrated in Figure 2(b). The figure shows that $\sigma$ increases almost linearly with the temperature which indicates an Arrhenius type thermally activated process. Linear shapes exhibited by all GPE signify that no phase transition was happening, which is the nature of amorphous polymer electrolytes. This deduced that the structural of GPE is stable over a wide temperature range. Referring to the $\sigma$ values, the conductivity rises with increasing temperature. This is strongly contributed by the rate of ion mobility. Ion mobility in this case is governed by the segmental motion of polymer which is caused by the expansion of polymer chains. As the temperature increases, polymer chain become more flexible and expanded, creating more free volumes (vacant sites). This leads the ionic species to undergo dipole arrangement by overcoming the activation energy to hop from one vacant site to other vacant sites and increases the ionic conductivity. 


\subsection{Dielectric relaxation studies}

(a)

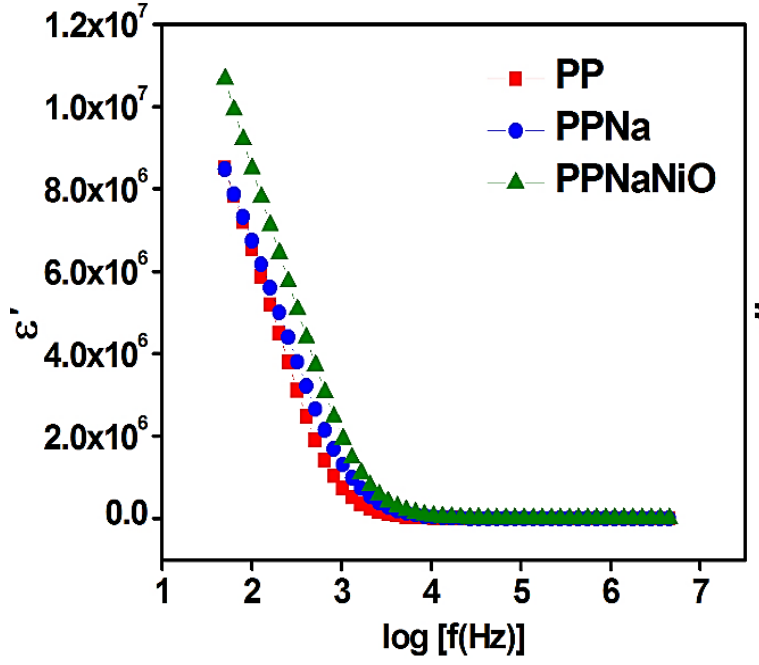

(b)

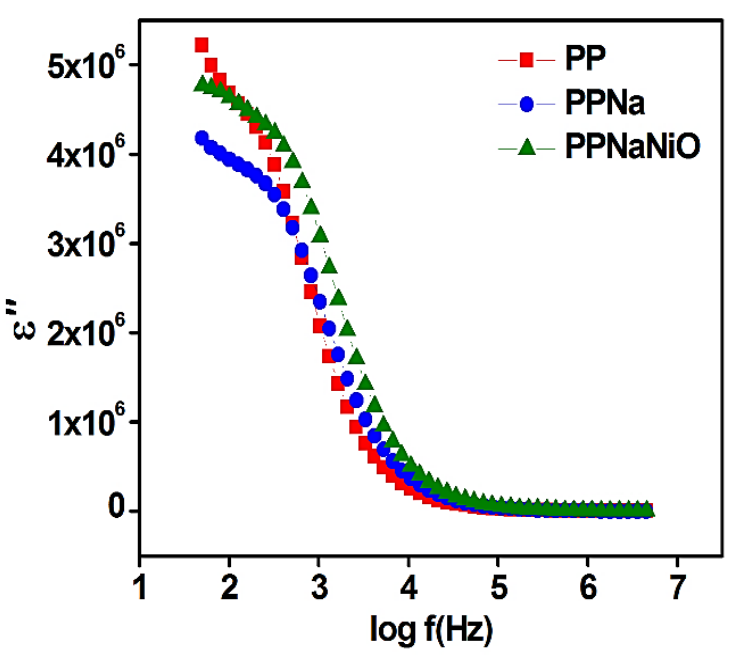

Figure 3. The frequency dependence of (a) $\varepsilon^{\prime}$ and (b) $\varepsilon^{\prime \prime}$ of PP, PPNa and PPNaNiO.

Dielectric analysis is substantial to investigate the transport of charge carriers in order to explain the polarizing ability of materials in the presence of an external oscillating electric field. This analysis can be presented in terms of the real and imaginary parts of complex permittivity (expressed in Equation 3) and is plotted against the frequency in the ranges $50 \mathrm{~Hz}$ to $5 \mathrm{MHz}$. .

$$
\varepsilon^{*}=\varepsilon^{\prime}-j \varepsilon^{\prime \prime}
$$

where $\varepsilon^{\prime}$ : real part of dielectric permittivity (dielectric constant) and $\varepsilon^{\prime \prime}$ : imaginary part of dielectric permittivity (dielectric loss). $\varepsilon^{\prime}$ is related to the capacitance and measures the alignment of dipoles and $\varepsilon^{\prime \prime}$ represents the loss of energy required to align the dipoles as heat energy. The value of $\varepsilon^{\prime}$ and $\varepsilon^{\prime \prime}$ at low frequencies is higher than the value at high frequency which indicates the dominance of electrode polarization. The electrode polarization is caused by the localized dipoles which responded to an externally applied electric field.
Based on Figure 3 (a), all dielectric spectra for PP, PPNa and PPNaNiO increase with decreasing frequency. This is because of at low frequency, ions get adequate time to response (via rotation, translation, and vibration) to the electric field, thus high number of dipoles present to contribute to electrode polarization (M.Y. Chong et.al., 2016). On the other hand, at high frequency, shorter time was given to the ions to align themselves along the field direction, therefore, lesser effect of electrode polarization and lower $\varepsilon^{\prime}$ values were observed. Likewise for Figure 3(b), larger $\varepsilon^{\prime \prime}$ values were obtained at low-frequency region. This indicates that internal heat is generated at this range of frequency. The heat generated because of the internal friction caused by de-acceleration of ions during the forward direction and acceleration of ions during the reverse direction (A. Arya et.al., 2017; V.S. Kumaran et.al., 2018). 
(a)

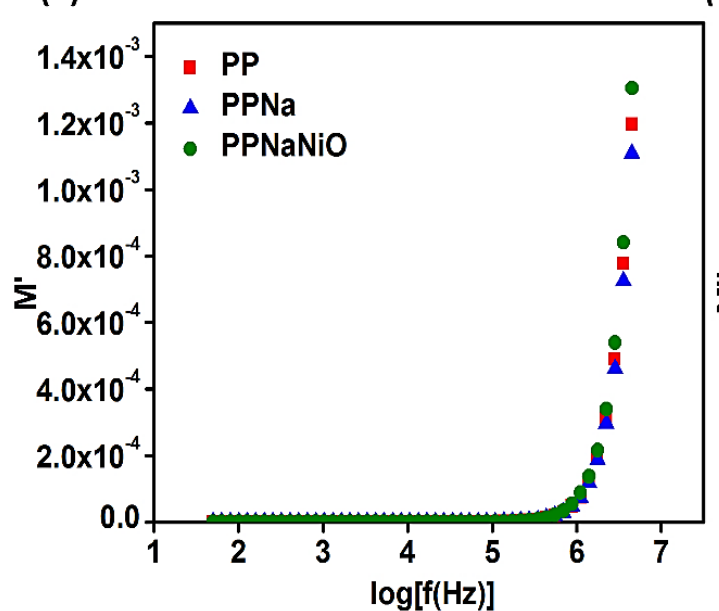

(b)

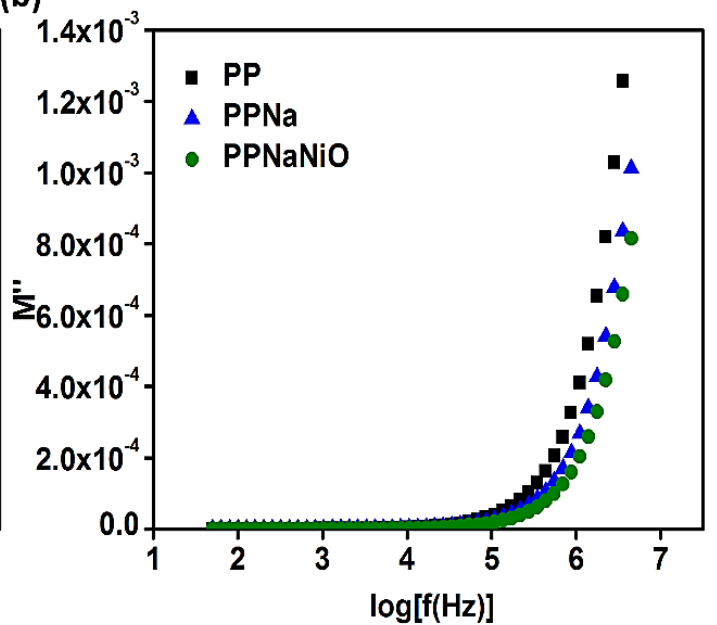

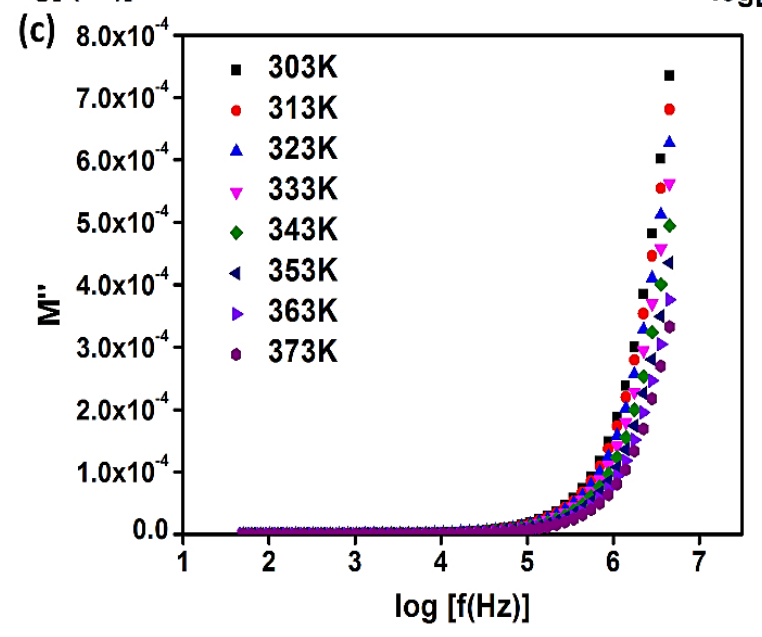

Figure 4. The plot of (a) $M^{\prime}$ and (b) $M^{\prime \prime}$ versus $\log$ f with samples of PP, PPNa and PPNaNiO. (c) The variation of $M^{\prime \prime}$ of PPNaNiO with frequency at different temperatures.

Electric modulus is useful to characterize the local dielectric relaxation in order to understand the electrode polarization effect. Basically, dielectric relaxation is caused by the lag in molecular polarization with respect to an oscillating electric field. Because of some polarizability mechanisms do not allow rapid reversal of the dipole alignment, electric modulus study is relatively important to complement the dielectric analysis as discussed in the previous section (C. Outline, 2015). The electric modulus is defined as the reciprocal of complex permittivity, hence, it can be expressed by the equation;

$$
M^{*}=M^{\prime}-j M^{\prime \prime}=\frac{1}{\varepsilon_{r}^{*}}=\frac{1}{\varepsilon^{\prime}-j \varepsilon^{\prime \prime}}
$$

Where $M^{\prime}$ and $M^{\prime \prime}$ are real and imaginary part of the complex electric modulus. In Figure 4(a), $M^{\prime}$ values approach zero at low frequencies which is attributed to the dominance of electrode polarization effect. Figure 4(b) depicts similar trend as $M^{\prime}$ whereby the dispersion relationship is only observed at high range of frequency. It is worth to be pointing out that, no resonance peak has been observed for all GPEs at higher frequency range due to the overall spectra were shifted to the right beyond the frequency window of the experiment (caused by the limitation of the instrument), thus, exhibiting the dispersion part. Generally, the increase of $M^{\prime}$ and $M^{\prime \prime}$ at high frequencies can be related to the resonance effect. When the frequency of the dipoles in the polymer systems matched with the frequency of 
the external field, the resonance dipolar relaxation is expected to occur. Figure 4(c) illustrates the variation of $M^{\prime \prime}$ with temperature for PPNaNiO. It can be noticed that the $M^{\prime \prime}$ values increases with the decrease of temperature and the curves were shifted towards higher frequency with the increase of temperature. Therefore, in overall, the dielectric relaxation at low temperature is large but decreases with increasing frequency. Thus, it can be concluded that the ion mobility in PPNaNiO is appreciable.

\subsection{DSSC performances}

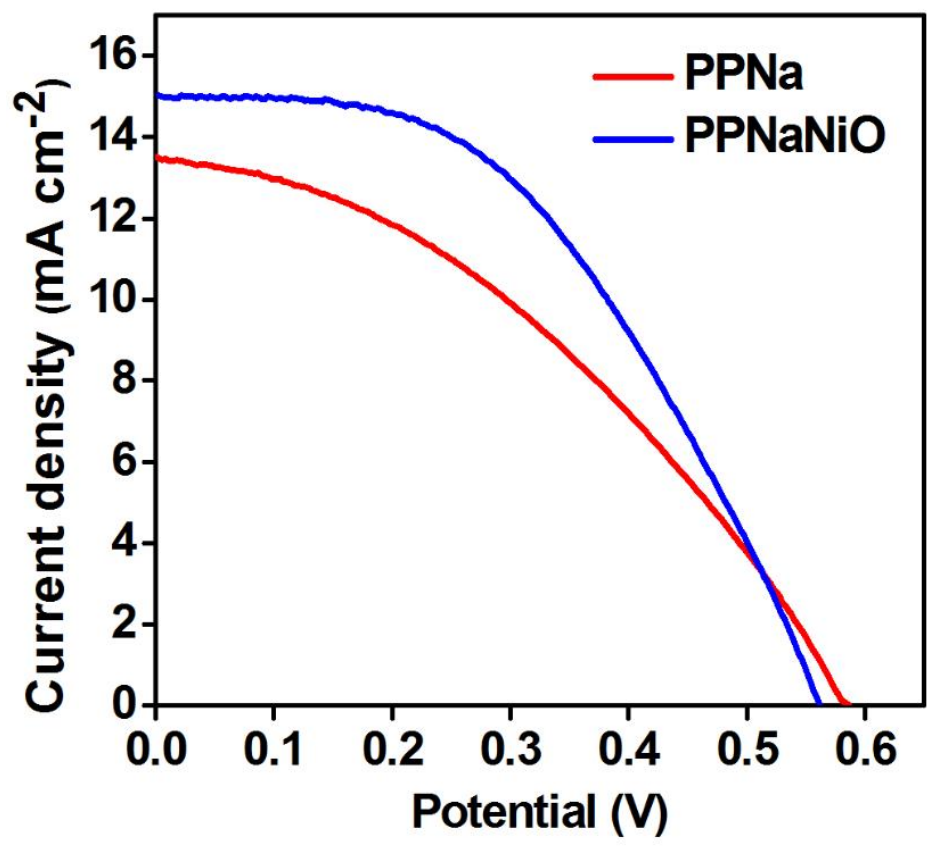

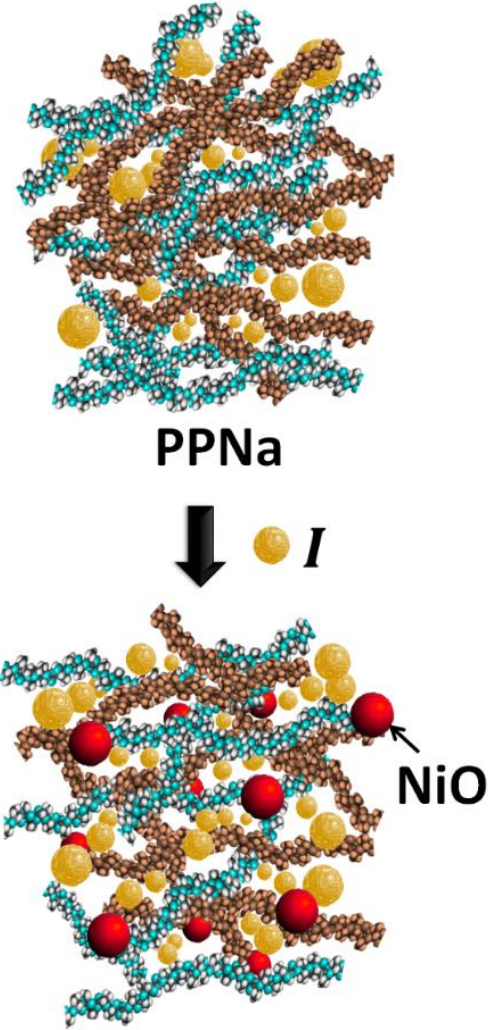

PPNaNiO

Figure 5. Graph of current density versus potential of the fabricated DSSCs employed with PPNa and PPNaNiO.

Figure 5 portrays the currentvoltage photovoltaic performance of DSSCs composed of $\mathrm{PPNa}$ and PPNaNiO under AM 1.5 illumination $\left(100 \mathrm{~mW} / \mathrm{cm}^{2}\right)$. The conversion

$$
\mathrm{FF}=(\operatorname{Vmax} \times \mathrm{Jmax}) /(\operatorname{Voc} \mathrm{x} \mathrm{Jsc})
$$$$
\eta(\%)=[(\operatorname{Vmax} \times \mathrm{J} \max ) / \operatorname{Pin}] \times 100 \%=[(\operatorname{Voc} \times \mathrm{Jsc} \times \mathrm{FF}) / \operatorname{Pin}) \times 100 \%
$$ 
Where Jsc is short-circuit current density $\left(\mathrm{mA} \mathrm{cm} \mathrm{cm}^{-2}\right.$, Voc is the open-circuit voltage $(\mathrm{V})$, Pin is the intensity of the incident light $\left(\mathrm{mW} \quad \mathrm{cm}^{-2}\right), \quad J m a x$ $\left(\mathrm{mA} \mathrm{cm}^{-2}\right)$ and $\mathrm{Vmax}(\mathrm{V})$ is photocurrent density and cell potential, respectively at the maximum power output. All parameters of current-voltage photovoltaic studies were summarized in Table 1. PPNaNiO has higher $\eta$ with the value of $3.98 \%$, which is $\sim 30.7 \%$ more than PPNa. It can be observed from the table that Jsc is the main contributing factor in determining the efficiency of the DSSCs. PPNaNiO has Jsc value of $15.03 \mathrm{~mA} \mathrm{~cm}^{-2}$, which is higher than PPNa with the Jsc value of $13.51 \mathrm{~mA}$ $\mathrm{cm}^{-2}$. The increase in Jsc value can be directly linked to the charge carrier and ionic mobility that influence the conductivity of the electrolyte as discussed in the previous section. NaI that was used as the dopant salt has a considerable low lattice energy. Low lattice energy is advantageous as $\mathrm{NaI}$ can easily be dissociated and produce more free ions for high efficiency of DSSC (A.K. Arof et.al., 2010). Upon the introduction of $\mathrm{NiO}$ into the GPE, the production of free ions were further augmented contributed from the ability of $\mathrm{NiO}$ to dissociate more $\mathrm{NaI}$ and lessen the interaction between the polymer chains (Q. Li et.al., 2014). This work represents the highest efficiency reported to-date for GPE as shown in Table 2.

Table 1. Parameter of current-voltage photovoltaic of the fabricated DSSC

\begin{tabular}{ccccc}
\hline Designation & Jsc $\left(\mathrm{mA} \mathrm{cm}^{-2}\right)$ & Voc $(\mathrm{V})$ & FF $(\%)$ & Efficiency, $\eta(\%)$ \\
\hline PPNa & 13.51 & 0.59 & 0.38 & 3.03 \\
PPNaNiO & 15.03 & 0.57 & 0.44 & 3.98 \\
\hline
\end{tabular}

Table 2. Literature Performance Parameter of DSSC

\begin{tabular}{ccccccc}
\hline Host polymer & Additive & $\begin{array}{c}J_{S C}(\mathrm{~mA} \\
\left.\mathrm{cm}^{-2}\right)\end{array}$ & $V_{O C}(\mathrm{~V})$ & FF (\%) & $\begin{array}{c}\text { Efficiency } \\
\eta(\%)\end{array}$ & Ref \\
\hline Agarose & LiI/ NiO & 6.20 & 0.63 & 0.52 & 2.02 & {$[18]$} \\
Agarose & LiI/ Co3O= & 3.82 & 0.69 & 0.44 & - & {$[19]$} \\
PAN & $\mathrm{MgI}_{2}$ & 6.82 & 0.68 & 0.54 & 2.56 & {$[20]$} \\
PAN & TPAI & 5.78 & 0.60 & 0.57 & 1.97 & {$[21]$} \\
P (VP-co-VAc) & TPAI & 6.86 & 0.73 & 0.62 & 3.07 & {$[22]$} \\
P (VP-co-VAc) & KI & 8.05 & 0.58 & 0.63 & 2.94 & {$[23]$} \\
P (VP-co-VAc) & THAI & 7.82 & 0.71 & 0.54 & 2.37 & {$[24]$} \\
\hline
\end{tabular}




\section{CONCLUSION}

The GPEs consisting PAN, $\mathrm{P}(\mathrm{VP}-\mathrm{co}-\mathrm{VAc}), \mathrm{NaI}$ with $\mathrm{NiO}$ additive were prepared and characterized. Through electrochemical study, PPNaNiO has improved the ionic conductivity from 2.33 to $3.54 \mathrm{mS} \mathrm{cm}^{-1}$. The increase in ionic conductivity is caused by the increase in ion movement across the amorphous structure of GPE contributed by the the $\mathrm{NaI}$ dissociation and the addition of $\mathrm{NiO}$. In addition, the conductivity of the GPEs were mainly contributed from the ionic motion which authenticated by Cole-Cole impedance plot and electrical modulus studies. As compared to the fabricated DSSC based PPNa, PPNaNiO exhibited an almost one time higher photoenergy conversion efficiency $(3.98 \%)$ with a short circuit current density of $15.03 \mathrm{~mA} \mathrm{~cm}$, open circuit voltage of $0.57 \mathrm{mV}$ and fill factor of $44.0 \%$.

\section{REFERENCES}

A.K. Arof, I.M. Noor, M.H. Buraidah, T.M.W.J. Bandara, M.A. Careem, I. Albinsson, et al., Polyacrylonitrile gel polymer electrolyte based dye sensitized solar cells for a prototype solar panel, Electrochim. Acta. 251 (2017) 223-234.

A. Arya, M. Sadiq, A.L. Sharma, Effect of variation of different nanofillers on structural, electrical, dielectric, and transport properties of blend polymer nanocomposites, Ionics (Kiel). (2017) 1 - 25.

A.K. Arof, M.H. Buraidah, L.P. Teo, S.R. Majid, R. Yahya, R.M. Taha, Characterizations of chitosanbased polymer electrolyte photovoltaic cells, Int. J. Photoenergy. 2010 (2010).
C. Outline, Ferroelectric and electrooptical properties of nano-glass ceramics, 2015.

H.C. Hassan, Z.H.Z. Abidin, M.A. Careem, A.K. Arof, Chlorophyll as sensitizer in $\mathrm{I}^{-} / \mathrm{I}_{3}^{-}$based solar cells with quasi-solid-state electrolytes, High Perform. Polym. 26 (2014) 647-652.

H.M. Ng, S. Ramesh, K. Ramesh, Exploration on the P(VP-co-VAc) copolymer based gel polymer electrolytes doped with quaternary ammonium iodide salt for DSSC applications: Electrochemical behaviors and photovoltaic performances, Org. Electron. Physics, Mater. Appl. 22 (2015) 132-139.

H.M. Ng, S. Ramesh, K. Ramesh, Efficiency improvement by incorporating 1-methyl-3propylimidazolium iodide ionic liquid in gel polymer electrolytes for dye-sensitized solar cells, Electrochim. Acta. 175 (2015) 169-175.

J. Wu, Z. Lan, J. Lin, M. Huang, Y. Huang, L. Fan, et al., Electrolytes in dye-sensitized solar cells, Chem. Rev. 115 (2015) 21362173.

L. Deanship, Effects of $\mathrm{TiO}_{2}$ and $\mathrm{TiC}$ Nanofillers on the Performance of Dye Sensitized Solar Cells based on the Polymer Gel Electrolyte of Cobalt Redox System, (2016).

M. Rasheduzzaman, P.B. Pillai, A.N.C. Mendoza, M.M. De Souza, A study of the performance of solar cells for indoor autonomous wireless sensors, in: 2016 10th Int. Symp. Commun. Syst. Networks Digit. Signal Process., 2016: pp. 1-6 
M.Y. Chong, A. Numan, C.-W. Liew, K. Ramesh, S. Ramesh, Comparison of the performance of copper oxide and yttrium oxide nanoparticle based hydroxylethyl cellulose electrolytes for supercapacitors, J. Appl. Polym. Sci. 134 (2016) 44636.

M.A.K.L. Dissanayake, C.A. Thotawatthage, G.K.R. Senadeera, T.M.W.J. Bandara, W.J.M.J.S.R. Jayasundara, B.-E. Mellander, Efficiency enhancement in dye sensitized solar cells based on PAN gel electrolyte with $\mathrm{Pr}_{4} \mathrm{NI}+\mathrm{MgI}_{2}$ binary iodide salt mixture, $\mathrm{J}$. Appl. Electrochem. 43 (2013) 891-901.

N.K.A. Hamed, M.K. Ahmad, N.S.T. Urus, F. Mohamad, N. Nafarizal, N. Ahmad, et al., Performance comparison between silicon solar panel and dye-sensitized solar panel in Malaysia, in: AIP Conf. Proc., 2017: p. 020029.

N.A.A. Latip, H.M. Ng, N. Farah, K. Ramesh, S. Ramesh, S. Ramesh, Novel development towards preparation of highly efficient ionic liquid based co-polymer electrolytes and its application in dye-sensitized solar cells, Org. Electron. Physics, Mater. Appl. 41 (2017) 33-41.

N. Duraisamy, A. Numan, S.O. Fatin, K. Ramesh, S. Ramesh, Facile sonochemical synthesis of nanostructured $\mathrm{NiO}$ with different particle sizes and its electrochemical properties for supercapacitor application, J. Colloid Interface Sci. 471 (2016) 136-144.
N.S. Ramesh, K.R. Pii, E. Acta, Efficiency improvement by incorporating 1-methyl-3propylimidazolium iodide ionic liquid in gel polymer electrolytes for dye-sensitized solar cells, Electrochim. Acta. 175 (2015) 169-175.

P.S. Dhapola, P.K. Singh, B. Bhattacharya, K. Surana, R. Mehra, M. Gupta, et al., Electrical, thermal, and dielectric studies of ionic liquid-based polymer electrolyte for photoelectrochemical device, High Perform. Polym. (2018) 095400831878021.

P. Nijisha, N.M. Bhabhina, S. Sindhu, Application of gel electrolyte in dye sensitized solar cells, Nanosyst. Physics, Chem. Math. 7 (2016) 752-754.

Q.-Y. Wu, X.-N. Chen, L.-S. Wan, Z.-K. $\mathrm{Xu}$, Interactions between Polyacrylonitrile and Solvents: Density Functional Theory Study and Two-Dimensional Infrared Correlation Analysis, J. Phys. Chem. B. 116 (2012) 83218330.

Q. Li, H. Ardebili, Atomistic investigation of the nanoparticle size and shape effects on ionic conductivity of solid polymer electrolytes, Solid State Ionics. 268 (2014) 156-161.

V.S. Kumaran, H.M. Ng, S. Ramesh, K. Ramesh, B. Vengadaesvaran, A. Numan, The conductivity and dielectric studies of solid polymer electrolytes based on poly ( acrylamide-co-acrylic acid ) doped with sodium iodide, (2018). 
V. Sundararajan, G. Selvaraj, H.M. Ng, S. Ramesh, K. Ramesh, C.D. Wilfred, et al., Exploring the effect of novel N-butyl-6methylquinolinium

bis(trifluoromethylsulfonyl)imide ionic liquid addition to poly(methyl methacrylate-comethacrylic) acid electrolyte system as employed in gel-state dye sensitized solar cells, Electrochim. Acta. 240 (2017) 361-370.
Y. Yang, J. Cui, P. Yi, X. Zheng, X. Guo, W. Wang, Effects of nanoparticle additives on the properties of agarose polymer electrolytes, J. Power Sources. 248 (2014) 988993.

Y. Yang, J. Gao, P. Yi, J. Cui, X. Guo, The influence of $\mathrm{Co}_{3} \mathrm{O}_{4}$ concentration on quasi-solid state dye-sensitized solar cells with polymer electrolyte, Solid State Ionics. 279 (2015) 1-5. 